\title{
Optimized Routing Model of Sensor Nodes in Internet of Things Network
}

\author{
Ximing Zhang, ${ }^{1 *}$ Jin Li, ${ }^{1}$ Rongfu Qiu, ${ }^{2}$ Tian-Shine Mean, ${ }^{3}$ and Fanzhu $\mathrm{Jin}^{3 * *}$ \\ ${ }^{1}$ China Southern Power Grid Company Limited, Guang Dong 510000, China \\ ${ }^{2}$ Digital Grid Research Institute, China Southern Power Grid, Guang Dong 510000, China \\ ${ }^{3}$ College of Mathematics and Information Engineering, Longyan University, Long Yan, Fujian 364000, China
}

(Received January 6, 2020; accepted June 16, 2020)

Keywords: Internet of Things, sensor, data acquisition, ant routing, path optimization

Heavy network traffic with a large number of sensor nodes causes high average energy consumption, a long average transmission delay, and a short network lifetime. To prevent this problem, we proposed and established an optimal path selection method for an Internet of Things (IoT) network based on an ant routing algorithm. The optimal path selection model considers the network lifetime and mobile distance, and analyzes node coverage conditions and communication energy consumption of all sensor nodes. The model is solved by an ant routing principle that finds the optimal path to a food source (a target node) with the maximum probability by considering the ant pheromone concentration (with a moving target node). We apply this principle to an IoT network. First, we divide a network monitoring area into several grids of the same size. Then, we repeat a process based on the principle until the network lifetime is greater than or equal to the time threshold to find the optimal path of a moving convergence node. Our simulation demonstrates that the optimal path model reduces the average energy consumption to reach the node, shortens the average transmission time to the node, and prolongs the network lifetime. The optimized routing model demonstrates a high data throughput and a stable operation.

\section{Introduction}

A wireless sensor network (WSN) is a group of autonomous electronic devices (sensors) in a network (sensor nodes) that collect data. WSNs process data such as temperature, humidity, light, noise, current, voltage, and electrical power. Wireless sensor nodes in a WSN transmit the data to gateway nodes through multihop routing and wireless transmission. The WSN then transmits the data from these gateway nodes to a user terminal for analysis and postprocessing. ${ }^{(1-3)}$ The performance of data acquisition technology in a WSN has a direct impact on accuracy, timeliness, energy consumption, and data postprocessing. ${ }^{(4-6)}$ For example, a huge amount of sensor data causes a sharp increase in power consumption. Rapid energy consumption leads to the premature failure of nodes due to their long running time. This changes the network

\footnotetext{
*Corresponding author: e-mail: zhangximing1980@163.com

** Corresponding author: e-mail: dennisbskim@hotmail.com https://doi.org/10.18494/SAM.2020.2796
} 
structure, shortens the lifecycle of the network, and reduces the application lifetime. ${ }^{(7-10)}$ A large number of wireless sensor nodes with different functions in the target monitoring area can solve this problem. However, sensors also have limited energy supply and computing power. This means that an energy-saving and efficient data acquisition/preprocessing technology is required to extend the life cycle of the network, but there are many problems in realizing such a technology. ${ }^{(11-13)}$ Many researchers have attempted to find ways of reducing energy consumption and extending the effective lifetime of a single node in WSNs. ${ }^{(14-16)}$ For example, to send one bit, a node of a wireless sensor consumes as much energy as that required for 1000 transmissions. Thus, the node of a sensor cannot send all its data. Instead, it uses an aggregation technology in the network to reduce energy consumption. Two types of aggregation technology are algebraic and quantile aggregation technologies. For example, aiming to decrease the energy consumption in a network, Liu et al. studied the continuous data of a curve query via aggregation algorithms. ${ }^{(17)}$

Data access in a WSN is different from that in a conventional Internet technology. In a conventional Internet technology, data is accessed through each IP address or domain name in a specific storage path. In contrast, a WSN obtains data as a whole packet from multiple nodes, not from a single specific node. ${ }^{(18)}$ In addition, wireless sensor nodes use gateway nodes when transmitting and gathering data to cache, fuse, and compute. They also process redundant and conflicting data to minimize the amount of data, and then finally transmit the data through specific gateway nodes to a user terminal. When one wireless sensor node perceives multiple targets, a data conflict is inevitable as the targets have different data owing to their different locations. However, a WSN allocates each node randomly but intensively and uses multiple nodes to monitor the same target at the same time. This reduces the data redundancy that may occur as a result of the multinode acquisition of data from the same target.

Traffic control is one of the applications of WSNs. Urban traffic congestion is becoming an increasingly serious problem in many countries, particularly because of air pollution. For example, the emission of pollutants by vehicles in Beijing, China, is responsible for more than half of the total air pollutants. Noise pollution is also a serious problem. Heavy traffic also causes high energy consumption and traffic accidents, both of which are increasing. ${ }^{(19)}$ It is expected that the use of the Internet of Things (IoT) to control traffic systems will help solve these problems. The Chinese government has recently proposed the use of the IoT for urban transportation systems. ${ }^{(20)}$ There have been several studies on the application of the IoT to traffic systems. Fortino et al. proposed an optimal path selection model (OPSM) based on the right-hand rule to improve the communication coverage of an IoT network. ${ }^{(21)}$ Particle swarm optimization is often used to find the optimal path of communication between sensor nodes. Gnawali et al. proposed and constructed an OPSM for an IoT network. They used the transfer probability of the Markov chain to set up efficient communication links and optimize a business flow and load balance. ${ }^{(2)}$ Mocanu et al. established an OPSM based on link information for an IoT network. Their model repeatedly switches links to locate a properly working sensor node to find an optimal path. ${ }^{(23)}$ These studies used OPSMs for an IoT network that can be successfully applied in a traffic control system to some extent. However, it is still needed to solve the problems of high energy consumption, a long transmission delay, and a short network lifetime 
when using the IoT to increase the efficiency of a traffic control system. We assume that these problems are caused by the influence of the network on finding the optimal path between sensor nodes. Thus, we propose an OPSM for an IoT network that uses an ant routing algorithm (ARA). An algorithm for the optimal setting of sensor nodes is established for the better utilization of sensors and a higher communication efficiency between sensor nodes, enabling the sensor networks to consume less energy in the nodes.

\section{Design of OPSM}

\subsection{Establishment of OPSM for sensors}

\section{Model assumptions}

(1) We selected an optimal path of sink nodes in a two-dimensional WSN in an intelligent IoT transportation system. Here, we make two assumptions: all sensor nodes in the network are randomly distributed in the monitoring area and the location of each sensor node remains unchanged. A sink node can be moved to any grid center in the monitoring area to collect data.

(2) A clustering algorithm can distribute the information from each location to different sink nodes and transform a path selection problem with multiple sink nodes into those with each sink node. It considers only the data from the sink nodes in a grid for a certain period of time.

(3) When each sensor node fails to communicate with sink nodes, the data from all sensor nodes is saved in a cache sequence. In this case, the nodes enter a 'sleeping state'. When each sensor node resumes communication with the sink nodes, each sensor node resumes a 'working state' to send its sensing data to the sink nodes through hop-by-hop communication.

(4) The energy of the sink nodes is unlimited, but that of each sensor node is limited and supplemented.

(5) A GPS module or another positioning method is used to obtain the location information of each sensor node.

(6) A unified energy consumption model is used.

\section{OPSM}

With the above assumptions, the IoT monitoring area is divided into $n \times n$ grids of equal size. All grids are uniformly coded from top to bottom and left to right. A sink node can be moved to any grid center to collect data, assuming that $V_{g}$ represents the collection of all grid centers after coding. $x_{n}$ is the status indicator of a sink node in a network center. $x_{n}=1$ when a sink node moves to grid center position $n$ to stay for a certain period of time to collect data and $x_{n}=0$ when it does not collect data. Then, the number of grid positions at which sink nodes need to dwell is defined as

$$
N_{n}=\sum_{n \in V_{g}} x_{n}
$$


If a sink node starts to collect data along a certain path from a grid center location and then returns to the initial grid center, the required time $t_{c}$ for a data collection cycle is defined as

$$
t_{c}=\sum_{n \in V_{m}} t_{c o l l}^{v} d_{T S P} / u
$$

Here, $t_{c o l l}^{v}$ is the dwell time of the sink node at the dwell grid location $v, V_{m}$ is the set of all dwell grid locations, $d_{T S P}$ is the shortest path for a sink node to move to a dwell grid location, and $u$ is the movement speed of the sink node.

To ensure that the data from the sink nodes can cover all the sensor nodes, the following conditions are met when each sensor node is set within the maximum range of a single hop distance of a dwell grid location:

$$
\exists v \in V_{m}, d_{i, v}<d_{\max }, \forall i \in V_{s},
$$

where $d_{i, v}$ is the distance between sensor node $i$ and sink node $v$ in the grid, $d_{\max }$ is the maximum distance in a single hop communication of the sensor node, and $V_{S}$ is a collection of sensor nodes in the IoT network.

In a data collection cycle, the energy consumption of a sensor node in a data transmission is defined as

$$
E t x=\sum_{v \in V_{i}} t_{c o l l}^{v}\left(f_{i, v} E_{\text {elec }}+f_{i, v} \varepsilon_{f s} d_{i, v}^{2}\right)
$$

$E_{\text {elec }}$ and $\varepsilon_{f s}$ are the amounts of electronic energy consumed by an amplifier when the node sends one bit of data and amplifies one bit of data, respectively. $f_{i, v}$ is the data transmission rate of node $i$ when a sink node dwells at grid location $v$. $V_{i}$ represents the set of all dwell grid positions where the communication distance to node $i$ is less than the maximum range of a single hop communication of the sensor node.

According to the above calculation, $E_{i}$ is the initial energy of sensor node $i$ in the network. $T_{\text {net }}$ indicates the IoT network lifetime. $T_{i}$, the lifetime of sensor node $i$, is

$$
T_{i}=\frac{E_{i} \sum_{v \in V_{i}} t_{\text {coll }}^{v}}{\sum_{v \in V_{i}} t_{\text {coll }}^{v}\left(f_{i, v} E_{\text {elec }}+f_{i, v} \varepsilon_{f s} d_{i, v}^{2}\right)}=\frac{E_{i}\left|V_{i}\right|}{\sum_{v \in V_{i}}\left(f_{i, v} E_{\text {elec }}+f_{i, v} \varepsilon_{f s} d_{i, v}^{2}\right)} .
$$

Here, $\left|V_{i}\right|$ is the number of elements in $V_{i}$ when a sink node has the same dwell time at each grid location. From these equations, the OPSM of the network lifetime is defined as follows:

$$
\max \min _{i \in V_{s}} T_{i} \text { s.t. } \exists v \in V_{m}, d_{i, v}<d_{\max }, \forall i \in V, x_{n} \in(0,1), n \in V_{g}
$$


To reduce the energy consumption for data transmission by sensor nodes and the data transmission delay, the model adopts the mobile distance of sink nodes. ${ }^{(16)}$ Then, Eq. (6) is modified to

$$
\max \left(\min _{i \in V_{s}} T_{i}\right) / d_{T S P} \text { s.t. } \exists v \in V_{m}, d_{i, v}<d_{\max }, \forall i \in V x_{n} \in(0,1), n \in V_{g} .
$$

\subsection{Model solution design for sensors based on ARA}

\section{Initialization of pheromone concentration}

According to the above OPSM, when ants initially select a mobile path, each adjacent node has the same probability of being selected. During an initialization, the ant pheromone concentration in the central area is set as $\tau$.

\section{Update rule of pheromone concentration of ants}

When an ant passes along a path $(u, v)$ in the model, the ant pheromone concentration on the path is updated according to the following equations.

$$
\begin{gathered}
c(u, v) \leftarrow(1-d) c(u, v)+h \cdot \Delta c(u, v), \\
\Delta c(u, v)=\frac{1}{\tau(u, v)}, \\
\tau(u, v)=\frac{t_{0}}{\tau}\left[1+\left(\frac{V(u, v)}{c(u, v)}\right)^{U}\right] .
\end{gathered}
$$

In these equations, $c(u, v)$ is the capacity of path $(u, v)$ and $d$ is the volatility coefficient of the concentration; $d \in(0,1) . h$ is a characteristic parameter related to the traffic status. $\tau(u, v)$ is the time for the ant to traverse from the start node (a source node) $(u, v)$ to the end node (a sink node). $t_{0}$ is the travel time along the path. $V(u, v)$ is the traffic of a section of the path. $U$ is a correction coefficient of the ant pheromone concentration. Through the above formula, the ant pheromone concentration is continuously optimized to obtain the maximum $c(u, v)$ for which the communication ability of the nodes in the network sensor is maximized.

\section{Global update of ant pheromone concentration}

When an ant arrives at a target node, it updates the pheromone concentration of each section (one node to the next node) from the source node to the target node according to the following equation:

$$
c(u, v) \leftarrow\left\{\begin{array}{l}
(1-d) c(u, v)+\Delta c(u, v),(u, v) \in p \\
(1-d) c(u, v),(u, v) \notin p
\end{array},\right.
$$


where $p$ is the maximum coefficient of volatilization of the ant pheromone concentration.

When an ant selects a path, the above ARA equations set a network dwell time and other parameters as Eq. (11). The steps of the OPSM with the ARA are as follows.

(1) In the ARA, ants start to search for the shortest path from a nest (a source node in the OPSM) to a food source (a sink node). Ants first stochastically try to find the shortest path to and from the food source. However, as an increasing number of ants select the shortest path to and from the source, pheromone accumulates on the path. Finally, the ants select the path with the highest pheromone concentration (the maximum probability of data transmission).

(2) To select the shortest path, ants need to decide which nodes to pass. They choose the next node (a target node) to move to. When the next node is not the food source (the sink node), they choose another node to go to in the shortest time and update the concentration of the pheromone provided by previous ants [Eq. (11)]. This step is repeated by the ants until the total travel time is greater than or equal to the threshold time from the nest to the food source [Eq. (10)].

\section{Results and Discussion}

To verify the performance of the OPSM with the ARA, we carried out experiments with the NS2 network simulation platform and compared the results with those of Cheng et al. and Galluzzi and Herman. ${ }^{(4,5)}$ Using the simulation conditions in Table 1, we selected the average energy consumption of the sensor nodes, the network lifetime, and the average transmission delay of data packets in the IoT network as indexes to evaluate performance in the simulations.

\section{Average energy consumption of sensor nodes in IoT network}

The average energy consumption of all sensor nodes in the IoT network for packet transmission is calculated as

$$
E_{\text {consumption }}=\frac{\sum_{i=1}^{R}\left(e_{i, \text { init }}-e_{i, \text { rest }}\right)}{l \times R} .
$$

Table 1

Simulation conditions.

\begin{tabular}{lc}
\hline Item & Description/specification \\
\hline Sensor nodes & randomly distributed in a rectangular area \\
\hline Number of sensor nodes & $50,100,150,200,250$ \\
\hline Maximum distance between sensor nodes & $50 \mathrm{~m}$ \\
\hline Communication protocol & $802.11 \mathrm{MAC}$ layer \\
\hline Communication bandwidth of sensor node & $1.8 \mathrm{Mb} / \mathrm{s}$ \\
\hline Data transmission interval & $2 \mathrm{~s}$ \\
\hline Packet load & 64 bytes \\
\hline Packet load of each sensor node & 32 bytes \\
\hline Power consumption of sensor node in idle time & $32 \mathrm{~mW}$ \\
\hline Energy consumption of sink node for each data packet & $\begin{array}{c}\text { for receiving } 420 \mathrm{~mW} \\
\text { for transmitting } 680 \mathrm{~mW}\end{array}$ \\
\hline
\end{tabular}


Here, $e_{i, \text { init }}$ is the initial energy information of sensor node $i, e_{i}$ is the remaining energy information of sensor node $i, R$ is the number of sensor nodes, and $l$ is the number of packets received by the target node. The above equation implies that sensor nodes have a higher performance with a smaller $E_{\text {consumption }}$.

Figure 1 shows that the average energy consumption of sensor nodes in our model (OPSM) is lower than that of the nodes in the studies of Wu et al. and Wang et al. ${ }^{(16,24)}$ It is natural that a system with more sensor nodes consumes more energy. However, the OPSM in this study consumed only about one-third of the energy of the other models regardless of the number of sensor nodes. This evidences that the OPSM of this study selects optimal paths with the initialization and update of paths, and balances the energy consumption of sensor nodes accordingly. The OPSM also prevents all sensor nodes from participating and thus reduces the energy consumption of the sensor nodes on subpaths efficiently. The OPSM performed very well in minimizing the average transmission delay of sensor node data packets as shown in Fig. 2.

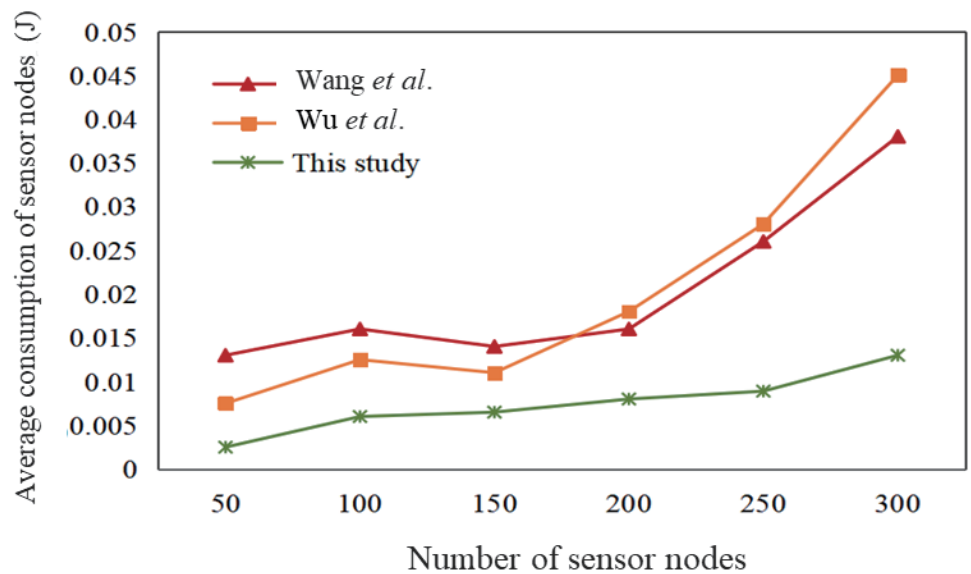

Fig. 1. (Color online) Comparison of average energy consumption of sensor nodes among different models.

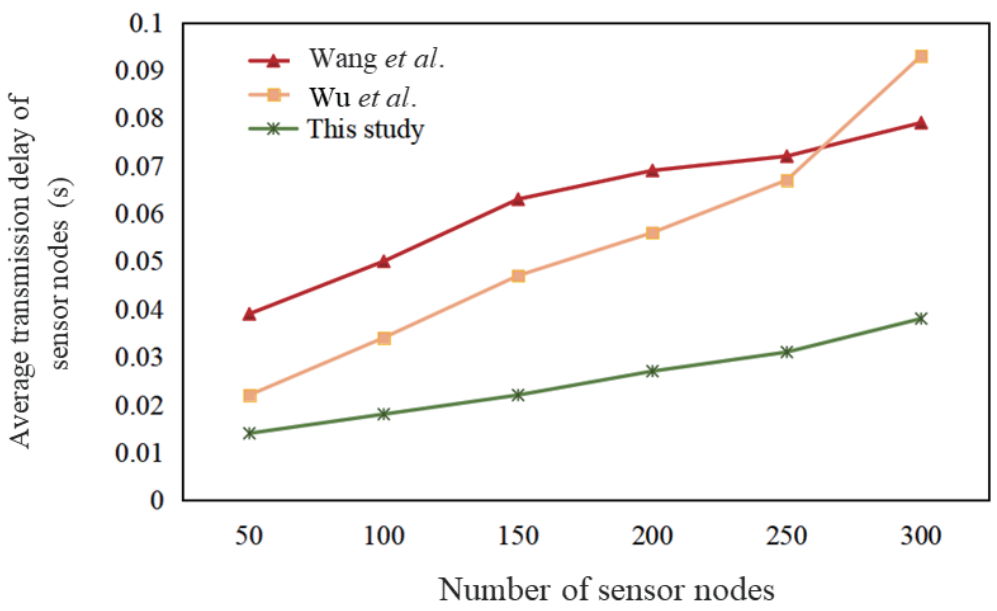

Fig. 2. (Color online) Comparison of average transmission delay of sensor nodes among different models. 
When the IoT network expands by increasing the number of sensor nodes, the average transmission delay increases. More sensor nodes increase the complexity of the network topology, increasing the number of communication transmissions from source nodes to target nodes. This increases the average transmission delay. Figure 2 shows that the OPSM has the shortest delay regardless of the number of sensor nodes. The delay of the OPSM is only about $25 \%$ of that in the model of Wang et al. ${ }^{(24)}$ and $33-50 \%$ of that in the model of Pan et al. ${ }^{(16)}$ The difference in delay between the OPSM and two other models increases with the number of sensor nodes. This is because the OPSM considers path selection with several single sink nodes, not with multiple sink nodes, but with the distance moved by the single sink node into the path-finding algorithm. This reduces the energy consumption of sensor nodes for data transmission and shortens the average transmission delay between the nodes.

The OPSM also has a longer network survival time than the other models. Figure 3 shows the network lifetimes of the three different models for different numbers of network sensor nodes. The results clearly demonstrate that the OPSM prolongs the network lifetime as the ARA helps it find the maximum data packet that can be transmitted along each path to select the optimal path. To study the stability and reliability of the proposed routing strategy, we simulated the transmission of the OPSM for different $\alpha$ and $\gamma$ values. $\alpha$ and $\gamma$ are parameters for the dependence of the data transmission capacity of each node and the distance of the transmission path. Figure 4 shows the statistical results. In addition to the order parameter, we measured the transmission performance of the OPSM using the intermediary centrality, the average transmission time $T_{a v g}$, the maximum transmission time $T_{\max }$, and the average path length $L_{\text {avg. }}$. The results show that $\alpha$ and $\gamma$ correspond to different network throughputs. When $\alpha>\gamma$, the network capacity decreases with increasing $\alpha$. When $\alpha \leq \gamma$, the transmission performance reaches a maximum and $\alpha$ and $\gamma$ remain stable. A phase transition also occurs when the network throughput $R$ is $20-25 \mathrm{bit} / \mathrm{s}$ (bps). The results clearly show that the OPSM has a higher overall performance than the other models. The OPSM has a shorter average transmission delay, a longer network life cycle, and less energy consumption with a more stable

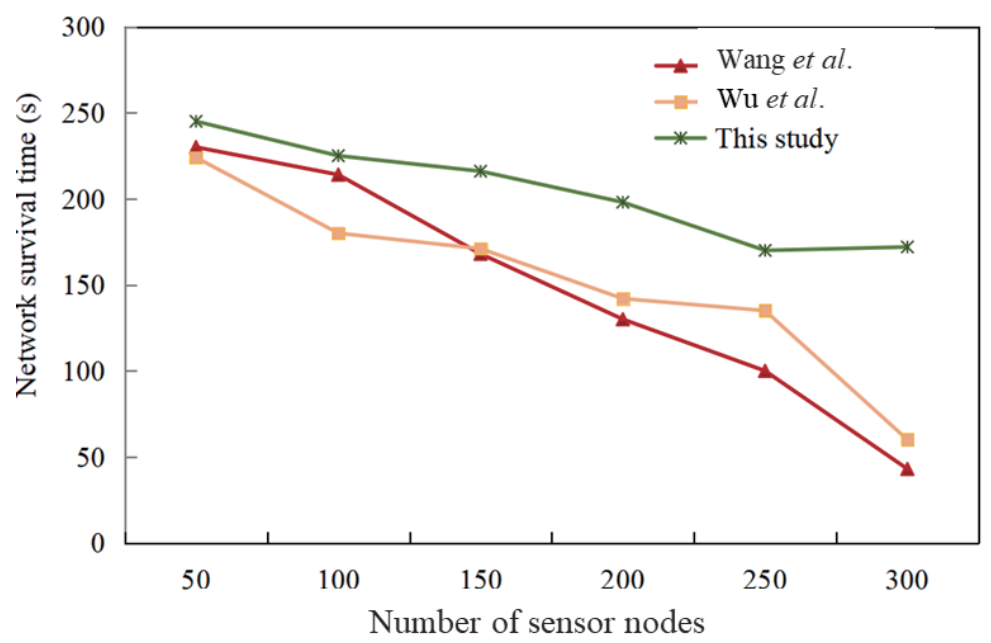

Fig. 3. (Color online) Comparison of network lifetime among different models. 


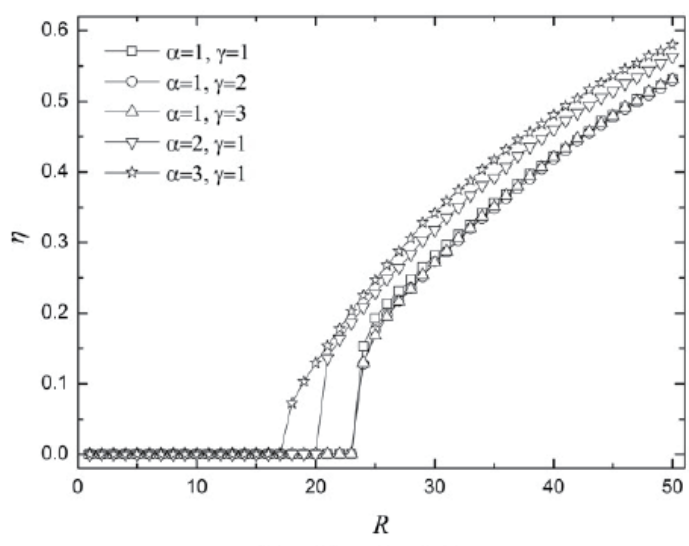

(a) $\eta$ versus $R$

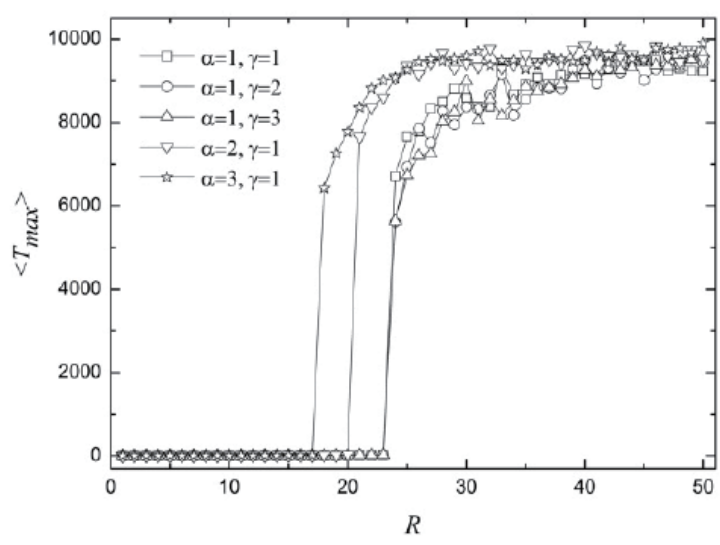

(c) $<T_{\max }>$ versus $R$

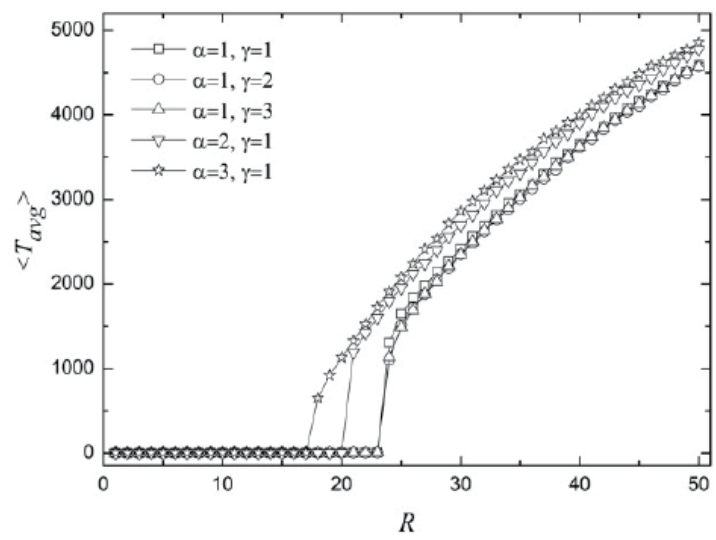

(b) $<T_{\text {avg }}>$ versus $R$

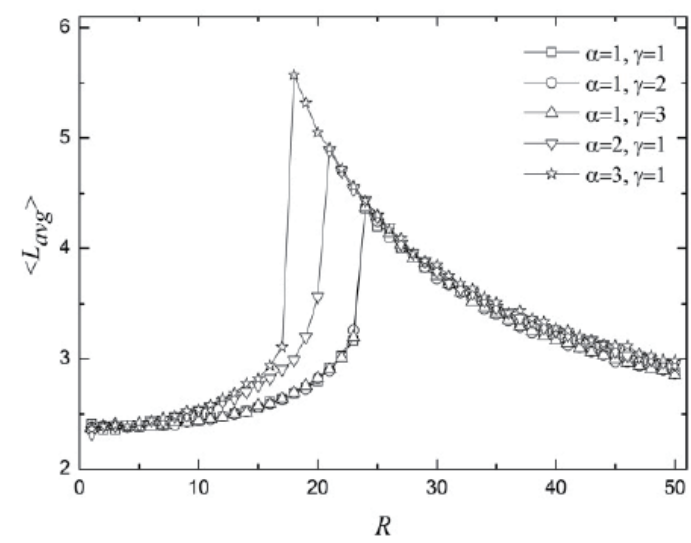

(d) $\left\langle L_{\text {avg }}>\right.$ versus $R$

Fig. 4. Simulation results of the routing model for network scale $N=100, m_{0}=4$, and node capacity $c=1$.

and reliable network performance. We integrated the ASA into the OPSM to meet the demands and standards of multisensor nodes in an IoT network. The ASA has been demonstrated to be very effective in optimizing the path of mobile convergence nodes. The OPSM newly proposed in this study is advantageous for many IoT applications.

\section{Conclusion}

We proposed and established an OPSM for IoT networks by integrating the ARA. IoT technologies require many sensors, generating demand for an efficient way to manage communication and save energy. The OPSM in this study validates itself to coordinate sensor nodes in an IoT environment very effectively, providing many benefits such as faster and uninterrupted communication, a longer network lifetime, less energy consumption, and stable performance. We expect that the OPSM will be used for urban traffic systems to reduce traffic congestion and accidents, suggesting that optimal paths for vehicles will also improve air quality. The application of the OPSM with the ASA in an IoT-based traffic control system should be examined in future research. 


\section{Acknowledgments}

This work was supported by China Southern Power Grid Co., Ltd. (Grant Nos. ZDKJXM20180009 and ZBKJXM20180347).

\section{References}

1 M. Viktor and K. Cukier: Big Data: A Revolution that Will Transform How We Live, Work and Think (Houghton Mifflin Harcourt, Boston, 2017) Chap. 1.

2 X. Li and H. Gong: Sci. Sinica Inf. 45 (2015) 1. https://doi.org/10.1360/N112014-00290

3 Z. He, Z. Cai, S. Cheng, and P. Chen: Theor. Comput. Sci. 607 (2017) 381. https://doi.org/10.1016/j.tcs.2015.07.056

4 S. Cheng, Z. Cai, and J. Li: IEEE Trans. Veh. Technol. 64 (2015) 5198. https://doi.org/10.1109/ TVT.2014.2375330

5 V. Galluzzi and T. Herman: Int. J. Distrib. Sens. N. 123 (2019) 82. https://doi.org/10.1155/2012/271860

6 I. Akyildiz, W. Su, Y. Sankarasubramaniam, and E. Cayirci: Comput. Netw. 38 (2016) 393. https://doi. org/10.1016/S1389-1286(01)00302-4

7 S. Xiao, S. Liu, M. Song, N. Ang, and H. Zhang: Multibody. Syst. Dyn. 48 (2020) 451. https://doi.org/10.1007/ s11044-019-09718-9

8 G. Fortino, A. Guerrieri, G. M. O Hare, and A. Ruzzelli: Comput. Appl. 35 (2018) 1934. https://doi.org/10.1016/ j.jnca.2012.07.016

9 Y. Zhang and J. Jiang: Annu. Rev. Control. 32 (2017) 229. https://doi.org/10.1016/j.arcontrol.2008.03.008

10 S. Ahmad, A. Lavin, S. Purdy, and Z. Agha: Neurocomputing 26 (2017) 134. https://doi.org/10.1016/ j.neucom.2017.04.070

11 M. Xie, S. Han, B. Tian, and S. Parvin: Comput. Appl. 34 (2018) 1302. https://doi.org/10.1016/j.jnca.2011.03.004

12 L. Akoglu, H. Tong, and D. Koutra: Knowl. Discov. 29 (2019) 626. https://arxiv.org/abs/1404.4679

13 D. Savage, X. Zhang, X. Yu, P. Chou, and Q. Wang: Soc. Network. 39 (2019) 62. https:// doi.org/10.1016/ Fj.socnet.2014.05.002

14 C. Phua, V. Lee, K. Smith, and R. Gayler: Inf. Fusion 35 (2019) 1517. https://arxiv.org/ftp/arxiv/ papers/1009/1009.6119.pdf

15 J. Bosman, G. Iacca, A. Tejada, H. J. Wörtche, and A. Liotta: Inf. Fusion 33 (2017) 41. https://doi.org/10.1016/ j.inffus.2016.04.007

16 P. Wu, X. Wu, G. Chen, M. Shan, and X. Zhu: Comput Comm. 83 (2016) 72. https://doi.org/10.1016/ j.comcom.2016.01.010

17 L. G. Liu, G. C. Luo, and K. Qin: Eurasip. J. Wirel. Comm. 38 (2017) 10. https://doi.org/10.1186/s13638-0160793-Z

18 Z. J. Duan, M. Y. Yan, and Z. P. Cai: Sensor 43 (2019) 481. https://doi.org/10.3390/s16040481

19 L. C. Zhang, Z. P. Cai, and J. L. Lu: Pers. Ubiquit. Comput. 19 (2018) 1111. https://doi.org /10.1007/s00779015-0880-x

20 A. Simitsis, and P. Vassiliadis: Decis. Supp. Syst. 45 (2018) 22. https://doi.org/10.1016/j.dss.2006.12.002

21 G. Fortino, A. Guerrieri, G. O' Hare, and A. Ruzzelli: Meas. Sci. Technol. 35 (2018) 1934. https://doi. org/10.1016/j.jnca.2012.07.016

22 O. Gnawali, R. Fonseca, K. Jamieson, M. Kazandjieva, D. Moss, and P. Levis: ACM Trans. Sens. Netw. 11 (2017) 168. https://doi.org/10.1145/2529988

23 D. C. Mocanu, E. Mocanu, P. H. Nguyen, M. Gibescu, and A. Liotta: Mach. Learn. 104 (2018) 243. https://doi. org/10.1007/s10994-016-5570-z

24 C. Wang, C. Luo, and H. W. Chen: Appl. Res. Comp. 33 (2018) 2462. 


\section{About the Authors}

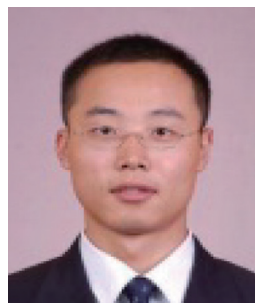

Ximing Zhang received his M.S. degree from Hunan University, China, in 2005. He works in the power dispatching control center of China Southern Power Grid Corporation. He holds a post in dispatching automation management. He is a professor-level senior engineer. His main research field is dispatching automation technology. (zhangximing1980@163.com)

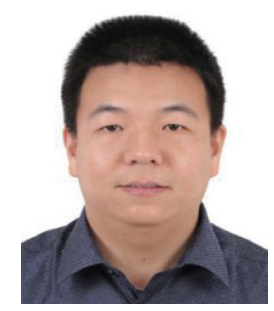

Jin Li received his M.S. degree from Central South University, China, in 2005. He works in the power dispatching and control center of China Southern Power Grid Corporation, and holds the title of professor-level senior engineer. His main research fields are power system communication protocol and power station automation technology and management.

(1ijin197908@163.com)

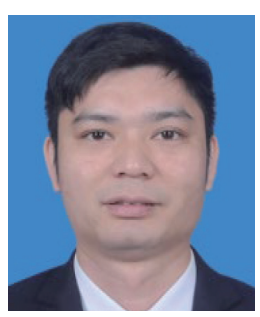

Rongfu Qiu received his M.S. degree from Shanghai Electric Power University, China, in 2011. He is a senior manager of China Southern Grid Digital Grid Research Institute Co., Ltd., with the title of engineer. His main fields of research are power dispatching automation systems and substation automation technology and management. (qiurongfu1984@163.com)

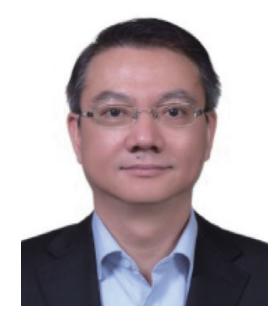

Tian-Shine Mean received his Ph.D. degree from National Central University, Taiwan, in 2001. He was an assistant professor and a director of an eCommerce center and an incubation center of National Kaohsiung University of Science and Technology in 2002-2006. Then, he has been working in various fields of business including aviation, fast moving consumer goods, information technology and banking. He is still collaborating with academicians to do research in Fintech.

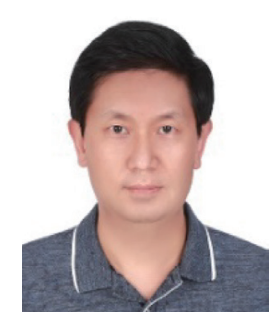

Fanzhu Jin received his Ph.D. degree from Seoul National University, Republic of Korea, in 2001. He then started working at Gdansk Medical University as an assistant professor. He is affiliated with the College of Mathematics and Information Engineering of Longyan University, Fujian, China. His research interests are in IoT and its applications. 